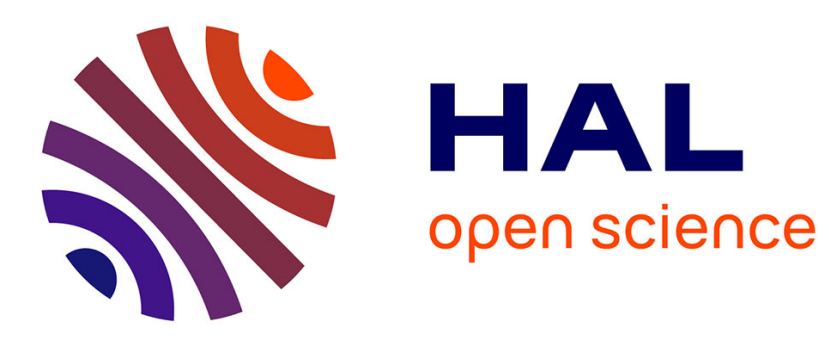

\title{
Interval impulsive observer for linear systems with aperiodic discrete measurements
}

Djahid Rabehi, Nacim Meslem, Adnen El Amraoui, Nacim Ramdani

\section{To cite this version:}

Djahid Rabehi, Nacim Meslem, Adnen El Amraoui, Nacim Ramdani. Interval impulsive observer for linear systems with aperiodic discrete measurements. IEEE Transactions on Automatic Control, 2021, 66 (11), pp.5407 - 5413. 10.1109/TAC.2020.3046126 . hal-02517009

\section{HAL Id: hal-02517009 \\ https://hal.science/hal-02517009}

Submitted on 24 Mar 2020

HAL is a multi-disciplinary open access archive for the deposit and dissemination of scientific research documents, whether they are published or not. The documents may come from teaching and research institutions in France or abroad, or from public or private research centers.
L'archive ouverte pluridisciplinaire HAL, est destinée au dépôt et à la diffusion de documents scientifiques de niveau recherche, publiés ou non, émanant des établissements d'enseignement et de recherche français ou étrangers, des laboratoires publics ou privés. 


\title{
Interval impulsive observer for linear systems with aperiodic discrete measurements
}

\author{
Djahid Rabehi, Nacim Meslem, Adnen El Amraoui, and Nacim \\ Ramdani, Member, IEEE
}

\begin{abstract}
This paper addresses the modeling and the design of an interval state observer for a linear timeinvariant plant in presence of sporadically available measurements corrupted by unknown-but-bounded errors and noise. The interval observer is modelled as an impulsive system where an impulsive correction is made whenever a measurement is available. The nonnegativity of the observation error between two successive measurements is preserved by applying the internal positivity based on the Müller's existence theorem, while at measurement times a linear programming constraint is added. A new methodology for designing the discrete-time observer gain is proposed that guarantees both nonnegativity and stability of the estimation error. The synthesis is performed by solving a set of Bilinear Matrix Inequalities (BMIs). The theoretical result is supported by numerical simulation.
\end{abstract}

\section{Index Terms}

Interval observers, LTI systems, sparse output measurements, hybrid system.

\section{INTRODUCTION}

The study of aperiodic sampled-data systems constitutes nowadays a very popular research topic in control. This is a consequence of the huge development of embedded and Networked

D. Rabehi, A and N. Ramdani are with the Université d'Orléans, INSA Centre Val de Loire (CVL), PRISME, EA 4229, Orléans, France. $\{$ djahid.rabehi, nacim.ramdani\}@univ-orleans.fr

N. Meslem is with the Université de Grenoble Alpes, CNRS, GIPSA-lab, F-38000 Grenoble, France. nacim.meslemegrenoble-inp.fr

A. El Amraoui is with the Université d'Artois, LGI2A, EA3926, F62400, Béthune, France. adnen.elamraouiduniv-artois.fr 
Control Systems (NCS), where sensor and control data are transmitted over digital communication channels. To reduce the communication load on these limited bandwidth channels, it is tempting to exchange data in an aperiodic manner. Besides, the presence of sampling jitters, packet dropouts and fluctuations in network accessibility further emphasize the interest in time-varying and aperiodic sampling $[1,2]$. In the context of state estimation, estimators for continuous-time systems with aperiodically sampled outputs have been studied under several frameworks; for instance, under the hybrid system framework [3] where the whole system is represented as an impulsive system [4], or under the time-delay system framework as a sampleddata system [5] where the system's output is held in between measurements.

This paper addresses the modeling and the design of an interval state observer, in a sense to be defined later, for a linear time-invariant plant in presence of sporadically available measurements perturbed by unknown-but-bounded errors and noise.

Interval observers are guaranteed state estimators in the sense that the existence of a solution can be verified and no solution can be lost. These observers have been introduced in [6] for continuous-time systems and extended to several classes of systems under the boundederror framework (see the survey [7]), whereas those for continuous-time systems with discrete measurements were firstly proposed in [8]. Basically, Interval observers compute trajectory tubes that are proven to contain the plant state trajectory while taking into account all uncertainties and disturbances acting on the plant and the measurements. The design of interval observers must ensure by construction the nonnegativity of the estimation error and its stability as well. Besides, the advantage of these observers compared to other guaranteed estimators, such as prediction-correction approaches $[9,10]$ for instance, is that the observer gain can be computed offline and the convergence of the estimation error can be proved a priori.

To be able to reconstruct a guaranteed state enclosure of the actual state for continuoustime linear systems in presence of sporadic discrete-time measurement, we propose an interval impulsive observer. The impulsive behavior is the result of the discrete nature of the measurements. In between two measurement time instants, the observer behaves as a continuous-time predictor based only on the evolution model. Then, at the measurement time instant, an impulsive correction adjusts the estimate state enclosure. Here, we consider that the inter-measurement time is unknown but belongs to an interval of time, contrariwise to [11] where it is chosen constant. The lower bound of the time interval is chosen in a way to avoid Zeno phenomenon (infinite number of samples in finite time). Both lower and upper bounds of the inter-measurement time 
are chosen a priori by the user, then our synthesis procedure tunes the observation gain to ensure both positivity and stability of the estimation error. The stability analysis of the bounds estimate is inspired by the work [4] while the positivity of the estimation error is ensured based on the internal positivity for dynamical systems as in [12], [13].

In the literature, the study of continuous interval observer with discrete measurements has been addressed in $[5,8,11]$. In [8], a continuous-discrete interval observer has been applied to a specific microalgae-based bioprocess. In [11], an analysis of an interval observer for continuoustime systems with discrete measurements has been proposed, where the measurement period is assumed constant. To the best of the authors' knowledge, there is no work dealing with the observation gain design in the context of continuous-discrete interval observation with sporadic discrete output.

The novelty of this paper resides in a new methodology for the design of the observer gain in presence of aperiodic measurements, that guarantees both positivity and stability of the interval estimation error. Moreover, the studied system includes both system perturbation and measurement noise while in [4] only output noise has been considered. The synthesis problem is formulated as a set of BMIs and Linear Programming (LP) constraints. An exponential convergence of the estimation error bounds is proved.

The paper is organized as follows. Some preliminaries are given in Section II. The stability verification of the interval observer for linear systems with aperiodic discrete measurements is performed in Section III. The proposed method for observer gain synthesis is detailed in Section IV. A numerical simulation is presented in Section V.

\section{PRELIMINARIES}

\section{A. Notations}

The set $\mathbb{R}, \mathbb{R}_{\geq}$and $\mathbb{N}$ are the set of real scalars, positive real scalars and nonnegative integers including zero, respectively. The induced matrix norm for a matrix $A \in \mathbb{R}^{n \times n}$ will be denoted as $\|\cdot\|$. Any $p \times m$ matrix whose elements are all ones or zeros are simply denoted by $\mathbb{1}_{p, m}$ or $\mathbf{0}$, respectively. $I_{p}$ denotes the identity matrix in $\mathbb{R}^{p \times p}$. Throughout this paper the inequality $A \geq B$ must be understood element-wise, for matrices as well as for vectors. $M=\max \{A, B\}$ is the matrix where each entry is $m_{i, j}=\max \left\{a_{i, j}, b_{i, j}\right\}$. Let us define $A^{+}=\max \{A, \mathbf{0}\}, A^{-}=A^{+}-A$; thus, $|A|=A^{+}+A^{-}$denotes the element-wise absolute value. A matrix $M \in \mathbb{R}^{n \times n}$ is said to be Metzler if all its off-diagonal entries are nonnegative. A symmetric matrix $P \in \mathbb{R}^{n \times n}$ is said 
to be negative (positive) definite if $v^{\top} P v<0(>0) \forall v \in \mathbb{R}^{n} \backslash\{0\}$ and it will be denoted by $P \prec 0(\succ 0)$. The Euclidean distance of $x \in \mathbb{R}^{n}$ to the closed set $\mathscr{A} \subset \mathbb{R}^{n}$ is denoted as $|x|_{\mathscr{A}}$ and is defined by $|x|_{\mathscr{A}}:=\inf _{y \in \mathscr{A}}\|x-y\|$. The symbol $\otimes$ refers to the Kronecker product.

\section{B. Definitions}

A scalar continuous function $\alpha(x)$, defined for $x \in[0, a)$ belongs to class $\mathscr{K}$ if it is strictly increasing and $\alpha(0)=0$. A function $\beta(s, t)$, defined for $s \in[0, a)$ and $t \in[0, \infty)$, is said to belong to class $\mathscr{K} \mathscr{L}$ if, (i) for each fixed $t \geq 0$, the mapping $\beta(s, t)$ belongs to class $\mathscr{K}$ with respect to $s$, (ii) for each fixed $s$, the mapping $\beta(s, t)$ is decreasing with respect to $t$ and $\lim _{t \rightarrow \infty} \beta(s, t) \rightarrow 0$.

In this paper we model the impulsive behaviour of the estimation error as a hybrid system. We consider the following formalism of hybrid systems introduced in [3]

$$
\begin{cases}\dot{x}=\mathscr{F}(x) & x \in \mathscr{C}, \\ x^{+}=\mathscr{G}(x) & x \in \mathscr{D},\end{cases}
$$

where $x \in \mathbb{R}^{n}$ is the state. $\mathscr{F}, \mathscr{C}, \mathscr{G}$ and $\mathscr{D}$ are the flow map, the flow set, the jump map and the jump set, respectively. $\mathscr{F}$ and $\mathscr{C}$ are supposed to be continuous, $\mathscr{G}$ and $\mathscr{D}$ are closed sets. The solutions to system (1) are defined on so-called hybrid time domains. A subset $\mathscr{E} \in \mathbb{R}_{\geq} \times \mathbb{N}$ is a compact hybrid time domain if $\mathscr{E}=\bigcup_{j=0}^{J-1}\left(\left[t_{j}, t_{j+1}\right], j\right)$ for some finite sequence of times $0=t_{0} \leq t_{1} \leq \cdots \leq t_{J}$. It is a hybrid time domains if for all $(T, J) \in \mathscr{E}, \quad \mathscr{E} \cap([0, T] \times\{0,1, \ldots, J\})$ is a compact hybrid domain. A function $\phi: \mathscr{E} \rightarrow \mathbb{R}^{n}$ is a hybrid arc if $\mathscr{E}$ is a hybrid time domain and if for each $j \in \mathbb{N}$, the function $t \mapsto \phi(t, j)$ is locally absolutely continuous on the interval $I^{j}=\{t:(t, j) \in \mathscr{E}\}$. In the sequel, the hybrid time domain $\mathscr{E}$ of the hybrid arc $\phi$ will be noted by $\operatorname{dom} \phi$. A hybrid arc $\phi$ is a solution to the hybrid system (1) if: (i) $\phi(0,0) \in \mathscr{C} \cup \mathscr{D}$; (ii) for all $j \in \mathbb{N}, \phi(t, j) \in \mathscr{C}$ and $\dot{\phi}(t, j)=\mathscr{F}(\phi(t, j))$ for almost all $t \in I^{j}$; (iii) for all $(t, j) \in \operatorname{dom} \phi$, such that $(t, j+1) \in \operatorname{dom} \phi, \phi(t, j) \in \mathscr{D}$ and $\phi(t, j+1)=\mathscr{G}(\phi(t, j))$. A solution $\phi$ to system (1) is maximal if it cannot be extended, complete if its domain, dom $\phi$, is unbounded. Also, it is Zeno if it is complete and $\sup _{t} \operatorname{dom} \phi<\infty$.

Definition 1 (Cooperative dynamics, [14]). A continuous-time linear system $\dot{x}(t)=A x(t)$ (discretetime linear system $x(t+1)=A x(t)$ ), with the state $x \in \mathbb{R}^{n}$ and $A \in \mathbb{R}^{n \times n}$, is said to be cooperative if $A$ is a Metzler (Nonnegative) matrix. 
The solutions of cooperative autonomous systems, initiated at $x\left(t_{0}\right) \geq 0$, stay nonnegative: $x(t) \geq 0$ for all $t \geq t_{0}$.

\section{INTERVAL IMPULSIVE OBSERVER ANALYSIS}

Let us consider the linear time invariant system of the form

$$
\dot{x}(t)=A x(t)+B u(t)+d(t), \quad \forall t \in \mathbb{R}
$$

where $x \in \mathbb{R}^{n}$ and $u \in \mathbb{R}^{m}$ are the state variables and the input of the system, and $d \in \mathbb{R}^{n}$ is system disturbance.

Let us consider the following discrete-time measurement equation

$$
y\left(t_{k}\right)=C x\left(t_{k}\right)+v\left(t_{k}\right), \quad \forall k \in \mathbb{N}
$$

where $y \in \mathbb{R}^{p}$ is the output of the system, and $v \in \mathbb{R}^{p}$ is the noise acting on the output measurement. It is assumed that there exist a minimal and maximal time between two consecutive measurement instants as follows.

Assumption 1. Let $\tau_{\max } \geq \tau_{\min }$ be two given real scalars that satisfy: $t_{k+1}-t_{k} \in\left[\tau_{\min }, \tau_{\max }\right] \forall k \in$ $\mathbb{N}$.

Based on the aperiodic discrete-time measurements, the goal here is to estimate an upper and a lower bound for the system state while ensuring the convergence of the estimation error. To do so, let us first introduce assumptions on the boundedness of the measurement noise and the system disturbance.

Assumption 2. Let a constant vector $\bar{v} \in \mathbb{R}_{\geq 0}^{p}$ be given such that $|v(t)| \leq \bar{v} \forall t \in \mathbb{R}_{\geq}$.

Assumption 3. Let two constant vectors $\underline{d}, \bar{d} \in \mathbb{R}^{n}$ be given such that $\underline{d} \leq d(t) \leq \bar{d}$ is satisfied $\forall t \in \mathbb{R}_{\geq}$.

Let us first define matrices $A^{M}=d_{A}+\left(A-d_{A}\right)^{+}, A^{N}=A^{M}-A$, where $d_{A}$ a diagonal matrix that contains only the diagonal elements of $A$, and $\bar{A}=\left[\begin{array}{cc}A^{M} & A^{N} \\ A^{N} & A^{M}\end{array}\right]$.

Assumption 4. Matrix $\bar{A}$ is non-singular.

Remark 1. In the sequel, we will adopt the notation of hybrid time domains, i.e., $\forall(t, j) \in \mathscr{E}$ : $x \equiv x(t, j)$ and $x^{+} \equiv x\left(t_{j}, j\right)$. 
The interval observer that we propose for system (2)-(3) has two steps;

First step: The interval observer behaves like an open-loop estimator between two successive measurement instants, i.e., $\forall(t, j) \in\left[t_{j}, t_{j+1}\right] \times\{j\}$

$$
\left\{\begin{array}{l}
\dot{x}=A^{M} \underline{x}-A^{N} \bar{x}+B u+\underline{d}, \\
\dot{\bar{x}}=A^{M} \bar{x}-A^{N} \underline{x}+B u+\bar{d}
\end{array}\right.
$$

In addition, the interval observer initial state at $(t, j)=\left(t_{0}, 0\right)$ satisfies the inclusion

$$
\underline{x}\left(t_{0}, 0\right) \leq x\left(t_{0}, 0\right) \leq \bar{x}\left(t_{0}, 0\right) .
$$

The dynamics of the estimation error during the inter-measurement time for both bounds $\underline{e}(t, j)=$ $x(t, j)-\underline{x}(t, j)$ and $\bar{e}(t, j)=\bar{x}(t, j)-x(t, j)$ can be obtained from equations (2) and (4) as

$$
\left[\begin{array}{c}
\dot{\dot{e}} \\
\dot{\bar{e}}
\end{array}\right]=\bar{A}\left[\begin{array}{l}
\underline{e} \\
\bar{e}
\end{array}\right]+\left[\begin{array}{l}
d-\underline{d} \\
\bar{d}-d
\end{array}\right]
$$

Let us denote by $\Xi(t, j)=\left[\begin{array}{l}d(t)-\underline{d} \\ \bar{d}-d(t)\end{array}\right]$ the second term of (6) (induced by the system disturbance), and $\bar{\Xi}=\left[\begin{array}{l}\bar{d}-\underline{d} \\ \bar{d}-\underline{d}\end{array}\right]$ its upper constant bound.

Note that, based on the construction of the matrices $A^{M}$ and $A^{N}$ as Metzler and nonnegative matrices, respectively, the matrix $\bar{A}$ is Metzler. In addition, the vector $\Xi(t, j)$ is nonnegative. Then, the solution to (6) is nonnegative which means that the lower and the upper bounds are nonnegative in the time interval $\left[t_{j}, t_{j+1}\right] \times\{j\}$ provided that their initial conditions are nonnegative, that is, the inclusion $\underline{x}\left(t_{j}, j\right) \leq x\left(t_{j}, j\right) \leq \bar{x}\left(t_{j}, j\right)$ is satisfied.

Second step: Using the output model (3), the system state at the measurement time instants can be presented as

$$
x^{+}=x+L(C x+v-y)
$$

introducing a fictitious reset equation that will help analyzing the reset dynamics of the estimation error. In Equation (7), $x^{+}=x\left(t_{j}, j\right)$ is the state vector right after the correction step, whereas $x=x\left(t_{j}, j-1\right)$ is the state vector right before the correction step. When the measurement is available, an impulsive correction of the estimated state enclosures will be done using the following correction equations

$$
\left\{\begin{array}{l}
\underline{x}^{+}=\left(I_{n}+L C\right)^{+} \underline{x}-\left(I_{n}+L C\right)^{-} \bar{x}-|L| \bar{v}-L y \\
\bar{x}^{+}=\left(I_{n}+L C\right)^{+} \bar{x}-\left(I_{n}+L C\right)^{-} \underline{x}+|L| \bar{v}-L y
\end{array}\right.
$$


where $L \in \mathbb{R}^{n \times p}$ is the observer gain to be designed.

From (7) and (8), the dynamics of the estimation error at measurement instants can be described by the following dynamical system

$$
\left[\begin{array}{l}
\underline{e}^{+} \\
\bar{e}^{+}
\end{array}\right]=\Gamma(L)\left[\begin{array}{l}
\underline{e} \\
\bar{e}
\end{array}\right]+\Upsilon
$$

where

$$
\Gamma(L)=\left[\begin{array}{ll}
\left(I_{n}+L C\right)^{+} & \left(I_{n}+L C\right)^{-} \\
\left(I_{n}+L C\right)^{-} & \left(I_{n}+L C\right)^{+}
\end{array}\right], \text {and } \Upsilon\left(t_{j}, j\right)=\left[\begin{array}{l}
|L| \bar{v}+L v\left(t_{j}, j\right) \\
|L| \bar{v}-L v\left(t_{j}, j\right)
\end{array}\right]
$$

The matrix $\Gamma(L)$ is an Internally Positive Realization of the state reset matrix $\left(I_{n}+L C\right)$. The positivity property of the reset matrix allows to preserve the order relation $\underline{x}(t, j) \leq x(t, j) \leq \bar{x}(t, j)$ after experiencing the reset (for more details about IPR for linear systems, see [13]).

Let us now consider the augmented error vector of the interval estimation as $\xi=\left[\underline{e}^{\top}, \bar{e}^{\top}\right]^{\top}$. Re-writing the system (6) and upper bounding its right-hand term using the upper bound of $\Xi(t, j)$, one gets $\forall(t, j) \in\left[t_{j}, t_{j+1}\right] \times\{j\}$

$$
\begin{aligned}
\dot{\xi} & =\bar{A} \xi+\Xi \\
& \leq \bar{A} \xi+\bar{\Xi}
\end{aligned}
$$

Now, using the results of the general comparison theorem [15] [16, Lemma 3.4] [17], the solution $\xi(t, j)$ of the system (10a) is upper bounded by the solution of the system

$$
\dot{\xi}_{u}=\bar{A} \xi_{u}+\bar{\Xi}
$$

provided that $\xi\left(t_{0}, 0\right) \leq \xi_{u}\left(t_{0}, 0\right)$, where $\xi_{u}(t, j) \in \mathbb{R}^{2 n}$ is an auxiliary variable that represents an upper bound of $\xi(t, j)$. Since Assumption 4 holds, the equilibrium of the system (11) is $\xi_{u e q}=-\bar{A}^{-1} \bar{\Xi}$. By introducing the shifted error $\xi_{0}=\xi_{u}-\xi_{u e q}$, the $\xi_{0}$-system dynamics is given as follows

$$
\dot{\xi}_{0}=\bar{A}\left(\xi_{0}+\xi_{\text {ueq }}\right)+\bar{\Xi}=\bar{A} \xi_{0}
$$

Remark 2. To simplify the stability analysis of the estimation error defined in (6) and (9), we will use the dynamics of the $\xi_{0}$-system. We justify our choice by the following reasons:

- The solution of the $\xi_{u}$-system in (11) is an upper bound of the solution of the $\xi$-system in (10a). Shifting the $\xi_{u}$-system by the value of its equilibrium $\xi_{u e q}$ using the $\xi_{0}$-system, the convergence of $\xi_{0}(t, j)$ to 0 means that $\xi_{u}(t, j)$ converges to $\xi_{u e q}$. 
- In the case of system (2) without state disturbance $(d(t)=0)$, the trajectories $\xi(t, j), \xi_{u}(t, j)$ and $\xi_{0}(t, j)$ are identical and have the same stability properties. To the contrary, when the state is under disturbance, then the trajectory $\xi(t, j)$ is upper bounded by the trajectory $\xi_{u}(t, j)$.

In the sequel, we will analyze the stability of the estimation error by analyzing the convergence to a set, that is ensuring that the distance to the set vanishes. Thus, with mild conditions, the stability analysis is straightforward under the hybrid system framework [3].

From equations (6) and (9) and by using the shifted upper bound of the estimation error (12), after adding the timer variable $\tau$, the hybrid system modeling the dynamics of the upper bound of the estimation error is given by

$$
\begin{cases}f\left(z_{0}\right)=\left[\begin{array}{c}
\bar{A} \xi_{0} \\
-1
\end{array}\right] & \forall z_{0} \in \mathscr{C} \\
g\left(z_{0}\right)=\left[\begin{array}{c}
\Gamma(L) \xi_{0}+\Upsilon_{2} \\
\mu
\end{array}\right] \quad \forall z_{0} \in \mathscr{D}\end{cases}
$$

where $\left.\left.\Upsilon_{2}\left(t_{j}, j\right)\right)=\Upsilon\left(t_{j}, j\right)\right)+(\Gamma(L)-I) \xi_{\text {ueq }}, z_{0}=\left[\xi_{0}^{\top}, \tau\right]^{\top}$ is the state variable of the hybrid system, $\mu \in\left[\tau_{\min }, \tau_{\max }\right]$ is the value of the timer $\tau$ after jump, and $\left[\tau_{\min }, \tau_{\max }\right]$ is the set of admissible values of the timer based on Assumption 1.

The flow and jump sets are defined as

$$
\begin{aligned}
& \mathscr{C}=\left\{\left(\xi_{0}, \tau\right) \in \mathbb{R}^{2 n} \times \mathbb{R}_{\geq} \mid \quad \tau \in\left[0, \tau_{\text {max }}\right]\right\} \\
& \mathscr{D}=\left\{\left(\xi_{0}, \tau\right) \in \mathbb{R}^{2 n} \times \mathbb{R}_{\geq} \mid \tau=0\right\} .
\end{aligned}
$$

It is worth noting that these sets do not force the system to jump until the timer violates the zero, then after the jump, the timer $\tau$ is reset to a value $\mu \in\left[\tau_{\min }, \tau_{\text {max }}\right]$.

Let us define the closed set $\mathscr{A}$ that contains all admissible values for the timer when the $\xi_{0}-$ system state is at the origin

$$
\mathscr{A}=\left\{z_{0}=\left(\xi_{0}, \tau\right) \in \mathbb{R}^{2 n} \times \mathbb{R}_{\geq} \mid \xi_{0}=0, \tau \in\left[0, \tau_{\text {max }}\right]\right\}
$$

Remark 3. As discussed in Remark 2, the stability of the $\xi_{0}$-system is sufficient for the stability of the $\xi$-system. Thus, if the $\xi_{0}$-system is stable relatively to $\mathscr{A}$, this implies that the $\xi$-system is practically stable relatively to $\mathscr{A}$.

Remark 4. The hybrid system (13) can be considered for the case without noise nor perturbation by omitting the term $\Upsilon_{2}\left(t_{j}, j\right)$. That is, if the system has $d(t, j)=0$ and $v\left(t_{j}, j\right)=0, \forall(t, j) \in$ 
$\operatorname{dom} \phi$, then we can pick $\underline{d}=\bar{d}=0$ and $\bar{v}=0$ which implies that $\bar{\Xi}=\mathbf{0}$, and $\Upsilon\left(t_{j}, j\right)=\mathbf{0} \forall(t, j) \in$ dom $\phi$. Thus we have $\Upsilon_{2}\left(t_{j}, j\right)=\mathbf{0}$.

We can now characterize the domain of the solutions of (13) when $\Upsilon_{2}\left(t_{j}, j\right)=0$. The variable $\tau$, acting as a timer, guarantees that for every initial condition $\phi(0,0) \in \mathscr{C} \cup \mathscr{D}$ the domain of every maximal solution $\phi$ to (13) when $\Upsilon_{2}\left(t_{j}, j\right)=0$ can be written as follows:

$$
\operatorname{dom} \phi=\bigcup_{j \in \mathbb{N}}\left(\left[t_{j}, t_{j+1}\right], j\right)
$$

with $\tau_{\min } \leq t_{j+1}-t_{j} \leq \tau_{\max }, \quad \forall j \in \mathbb{N} \backslash\{0\}$. Furthermore, assuming $t_{0}=0$, the structure of the above hybrid time domain implies that for each $(t, j) \in \operatorname{dom} \phi$ we have

$$
t \leq(j+1) \tau_{\max }
$$

Equation (16) will play a key role in establishing GES of the set $\mathscr{A}$ for hybrid system (13) when $\Upsilon_{2}\left(t_{j}, j\right)=\mathbf{0}$.

Let us now recall the definitions of the global exponential stability (GES) and the input-to-state stability (ISS) of closed sets for a general hybrid system.

Definition 2. ([18], [19]) Let $\mathscr{A} \subset \mathbb{R}^{n_{\phi}}$ be closed. The set $\mathscr{A}$ is said to be:

- GES for the hybrid system (13) when $\Upsilon_{2}\left(t_{j}, j\right)=\mathbf{0}$ if there exist $\lambda, \kappa \in \mathbb{R}_{>}$such that every solution $\phi$ to (13) when $\Upsilon_{2}\left(t_{j}, j\right)=\mathbf{0}$ satisfies:

$$
\forall(t, j) \in \operatorname{dom} \phi, \quad|\phi(t, j)|_{\mathscr{A}} \leq \kappa e^{-\lambda(t+j)}|\phi(0,0)|_{\mathscr{A}}
$$

- ISS for (13) with respect to $\Upsilon_{2}$ if there exist $\beta \in \mathscr{K} \mathscr{L}$ and $\alpha \in \mathscr{K}$ such that every solution $\phi$ to (13) satisfies:

$$
\forall(t, j) \in \operatorname{dom} \phi
$$

$$
|\phi(t, j)|_{\mathscr{A}} \leq \max \left\{\beta\left(|\phi(0,0)|_{\mathscr{A}}, t+j\right), \alpha\left(\left\|\Upsilon_{2}\right\|_{(t, j)}\right)\right\}
$$

Before setting our first result in the next theorem, let introduce the following lemma.

Lemma 1 ([4]). Let $\rho$ be a strictly negative real number. Choose $\gamma \in\left(0, \frac{|\rho|}{1+\tau_{\max }}\right]$ and $\omega \in$ $\left[\frac{\tau_{\max }|\rho|}{1+\tau_{\max }}, \infty\right)$. Let $\phi$ be any solution to the hybrid system (13). Then for every $(t, j) \in \operatorname{dom} \phi$, one has $\rho j \leq \omega-\gamma(t+j)$. 
The idea behind using Lemma 1 is to allow for the Lyapunov function to increase locally. This increase is compensated by instantaneous decrease at jumps which renders the overall hybrid dynamics stable. This stability property is the main result of [3, Proposition 3.29].

Theorem 1. Let Assumptions 1,2, 3, and 4 hold. For a given gain matrix $L \in \mathbb{R}^{n \times p}$, if there exist a symmetric positive definite matrix $P \in \mathbb{R}^{2 n \times 2 n}$ such that

$$
\Gamma(L)^{\top} e^{\bar{A}^{\top} \mu} P e^{\bar{A} \mu} \Gamma(L)-P \prec 0 \quad \forall \mu \in\left[\tau_{\min }, \tau_{\max }\right]
$$

is satisfied, then the hybrid system (13)-(14) is Input-to-State-Stable (ISS) with respect to the set $\mathscr{A}$ defined in (15). Thus, the system (4), (8) is an interval observer for the system (2)-(3) with ISS estimation error relatively to $\mathscr{A}$ provided that $\underline{x}\left(t_{0}, 0\right) \leq x\left(t_{0}, 0\right) \leq \bar{x}\left(t_{0}, 0\right)$. Moreover, if $d(t, j)=0$ $\forall(t, j) \in \operatorname{dom} \phi$ in (2) and $v\left(t_{j}, j\right)=0 \forall(t, j) \in$ dom $\phi$ in (3), then the interval observer (4), (8) for the system (2)-(3) has a globally exponentially stable (GES) estimation error relatively to $\mathscr{A}$.

The proof of stability in Theorem 1 follows the main lines of the proof of [4, Theorem 1] with appropriate modifications due to the fact that we study the stability of an upper-bound of the interval estimation error. These modifications have a double role. First, they have to guarantee the order preserving property for the estimation error. Then, they manage the effect of the state disturbance.

Proof of Theorem 1. The proof is split into two parts; studying the nonnegativity of the estimation error, then the stability of the upper bound of the estimation error.

The cooperativity of the estimate error: Based on Assumption 3 and due to the Metzler property of the matrix $\bar{A}$ of (6), the flow dynamics (6) is nonnegative. On the other hand, the observer updates the estimates at measurement times by means of (8), where its estimation error is represented by the jump dynamics (9). The matrix $\Gamma(L)$ is structurally nonnegative while Assumption 2 ensures the nonnegativity of the vector $\Upsilon\left(t_{k}\right)$. Consequently, the estimation error preserves the order relation $\underline{x}\left(t_{j}, j\right) \leq x\left(t_{j}, j\right) \leq \bar{x}\left(t_{j}, j\right)$ after experiencing the reset. Finally, with the initial condition given as $\underline{x}\left(t_{0}, 0\right) \leq x\left(t_{0},, 0\right) \leq \bar{x}\left(t_{0}, 0\right)$, the errors $\underline{e}(t, j)$ and $\bar{e}(t, j)$ of $(13)$ are nonnegative for all $(t, j) \in \operatorname{dom} \phi$.

The stability of the estimation error upper-bound: Instead of studying the stability of $z=\left[\xi^{\top}, \tau\right]^{\top}$, we use its shifted upper bound trajectory $z_{0}=\left[\xi_{0}^{\top}, \tau\right]^{\top}$ as discussed in Remark 2. Let us consider 
the Lyapunov function candidate for the hybrid system (13)-(14) defined $\forall z_{0} \in \mathbb{R}^{2 n} \times \mathbb{R}_{\geq}$

$$
V\left(z_{0}\right)=\xi_{0}^{\top} e^{\bar{A}^{\top} \tau} P e^{\bar{A} \tau} \xi_{0}
$$

Note that there exist two positive scalars $l_{1}, l_{2}$ such that

$$
l_{1}\left|z_{0}\right|_{\mathscr{A}}^{2} \leq V\left(z_{0}\right) \leq l_{2}\left|z_{0}\right|_{\mathscr{A}}^{2} \forall z_{0} \in \mathscr{C} \cup \mathscr{D} \cup \mathscr{G}(\mathscr{D})
$$

Due to the positive definiteness of $P$ and the non-singularity of the matrix $e^{\bar{A}}$ for every $\tau$, by continuity arguments, one can set

$$
l_{1}=\min _{\tau \in\left[0, \tau_{\max }\right]} \lambda_{\min }\left(e^{\bar{A}^{\top} \tau} P e^{\bar{A} \tau}\right), \quad l_{2}=\max _{\tau \in\left[0, \tau_{\max }\right]} \lambda_{\max }\left(e^{\bar{A}^{\top} \tau} P e^{\bar{A} \tau}\right)
$$

where $\lambda_{\min }(\cdot)$ and $\lambda_{\max }(\cdot)$ denote the smallest and the largest eigenvalue of their matrix argument, respectively. From (20) we can find

$$
\nabla V\left(z_{0}\right)=\left(2 e^{\bar{A}^{\top} \tau} P e^{\bar{A} \tau} \xi_{0}, \xi_{0}^{\top} \bar{A}^{\top} e^{\bar{A}^{\top} \tau} P e^{\bar{A} \tau} \xi_{0}+\xi_{0}^{\top} e^{\bar{A}^{\top} \tau} P \bar{A} e^{\bar{A} \tau} \xi_{0}\right)
$$

For simplicity, throughout this proof, we write $\Gamma$ and $\Upsilon_{2}$ instead of $\Gamma(L)$ and $\Upsilon_{2}\left(t_{j}, j\right)$, respectively. From system (13)-(14), and under the fact that $e^{\bar{A} \mu}$ and $\bar{A}$ commute, the variation of the Lyapunov function over the inter-sampling time is then $\left\langle\nabla V\left(z_{0}\right), f\left(z_{0}\right)\right\rangle=0 \forall z_{0} \in \mathscr{C}$. Thus, the stability of the upper-bound of the error dynamics can be verified by considering the evolution of the Lyapunov function for every $z_{0} \in \mathscr{D}$. In addition, whenever $z_{0} \in \mathscr{D}$, we have $\tau=0$, which implies

$$
\begin{aligned}
& V\left(g\left(z_{0}\right)\right)-V\left(z_{0}\right)=\left(\Gamma \xi_{0}+\Upsilon_{2}\right)^{\top} e^{\bar{A}^{\top} \mu} P e^{\bar{A} \mu}\left(\Gamma \xi_{0}+\Upsilon_{2}\right)-\xi_{0}^{\top} P \xi_{0} \\
&=\xi_{0}^{\top}\left(\Gamma e^{\bar{A}^{\top} \mu} P e^{\bar{A} \mu} \Gamma-P\right) \xi_{0}+2 \Upsilon_{2}^{\top} e^{\bar{A}^{\top} \mu} P e^{\bar{A} \mu} \Gamma \xi_{0} \\
&+\Upsilon_{2}^{\top} e^{\bar{A}^{\top} \mu} P e^{\bar{A} \mu} \Upsilon_{2}
\end{aligned}
$$

GES stability: First, we discuss the case of a system without noise nor perturbation, i.e., when $v\left(t_{j}, j\right)=0$ and $d(t, j)=0$, which implies $\Upsilon_{2}=\mathbf{0}$. Recall that $\xi_{0}^{\top} \xi_{0}=\left|z_{0}\right|_{\mathscr{A}}^{2}$, based on inequality (19) there exists a small enough $\eta \in \mathbb{R}_{>}$such that (24) becomes

$$
V\left(g\left(z_{0}\right)\right)-V\left(z_{0}\right) \leq-\eta \xi_{0}^{\top} \xi_{0}=-\eta\left|z_{0}\right|_{\mathscr{A}}^{2} \quad \forall z_{0} \in \mathscr{D}
$$

Without loss of generality, let us assume that $l_{2}$ in (22) and $\eta$ in (25) satisfy $1-\frac{\eta}{l_{2}}>0$. Define $\theta_{1}=\ln \left(1-\frac{\eta}{l_{2}}\right)$ and observe that $\theta_{1}<0$. Hence

$$
V\left(g\left(z_{0}\right)\right) \leq e^{\theta_{1}} V\left(z_{0}\right) \quad \forall z_{0} \in \mathscr{D} .
$$


Consider that $\phi$ is a maximal solution to (13)-(14). As proven in [3, Proposition 3.29 ], and similarly to [4, Theorem 1], using Lemma 1 one can find that the solution $\phi$ satisfies inequality (17) in Definition 2. Thus, the set $\mathscr{A}$ defined in (15) is GES for the hybrid system (13)-(14). Based on Remark 3, one can conclude that the interval observer (4), (8) with the system (2)-(3) have a GES estimation error relatively to $\mathscr{A}$.

ISS stability: Returning to the general case where $\Upsilon_{2} \neq 0$. We apply Young's inequality $2 a^{\top} b \leq$ $\frac{\eta}{2} a^{\top} a+\frac{2}{\eta} b^{\top} b$ where $a=\xi_{0}$ and $b^{\top}=\Upsilon_{2}^{\top} e^{\bar{A}^{\top} \mu} P e^{\bar{A} \mu} \Gamma$ on the right hand part of equation (24). Then, we get

$$
\begin{aligned}
V\left(g\left(z_{0}\right)\right)-V\left(z_{0}\right) \leq & -\frac{\eta}{2} \xi_{0}^{\top} \xi_{0} \\
& +\Upsilon_{2}^{\top}\left[e^{\bar{A}^{\top} \mu}\left(\frac{2}{\eta} P e^{\bar{A} \mu} \Gamma \Gamma^{\top} e^{\bar{A}^{\top} \mu}+I\right) P e^{\bar{A} \mu}\right] \Upsilon_{2} \\
\leq & -\frac{\eta}{2 l_{2}} V\left(z_{0}\right)+\Upsilon_{2}^{\top} \Upsilon_{2}\left\|\frac{2}{\eta} P e^{\bar{A} \mu} \Gamma \Gamma^{\top} e^{\bar{A}^{\top} \mu}+I\right\| \\
& \times\|P\| \max _{\mu \in\left[\tau_{\min }, \tau_{\max }\right]}\left(\left\|e^{\bar{A} \mu}\right\|^{2}\right)
\end{aligned}
$$

From (19), we have $\left\|\frac{2}{\eta} P e^{\bar{A} \mu} \Gamma \Gamma^{\top} e^{\bar{A}^{\top} \mu}+I\right\| \cdot\|P\| \leq\left(\frac{2}{\eta}\|P\|+1\right)\|P\|$. In system (13), we have $\Upsilon_{2}=\Upsilon+(\Gamma-I) \xi_{\text {ueq }}$. Using the triangle inequality, one obtain

$$
\Upsilon_{2}^{\top} \Upsilon_{2} \leq 2 \Upsilon^{\top} \Upsilon+2\left\|\xi_{\text {ueq }}\right\|^{2}\|(\Gamma-I)\|^{2}
$$

where the uncertain term $\Upsilon$ is bounded by $2|L| \bar{v}$, provided that $v\left(t_{j}, j\right)$ is essentially bounded. The vector function $\Upsilon_{2}$ defined in (9) satisfies $\Upsilon_{2}^{\top} \Upsilon_{2}=2|||L| \bar{v}\left\|^{2}+2\right\| L v\left\|^{2} \leq 4|||L| \bar{v}\right\|^{2}$, and based on Assumption 3 we thus have $\Upsilon_{2}^{\top} \Upsilon_{2} \leq\left. 4\||| L\|\right|^{2} \sup _{t}\|v\|_{(t, j)}^{2}$. Consequently, by replacing these inequalities in (27), the Lyapunov function at jumps satisfies

$$
V\left(g\left(z_{0}\right)\right) \leq e^{\theta_{2}} V\left(z_{0}\right)+\zeta_{1} \sup _{t}\|v\|^{2}+\zeta_{2} \quad \forall z_{0} \in \mathscr{D}, v \in \mathbb{R}^{p}
$$

where $\theta_{2}=\ln \left(1-\frac{\eta}{2 l_{2}}\right)$ is a negative scalar while $\zeta_{1}$ and $\zeta_{2}$ are positive scalars defined as follows

$$
\begin{gathered}
\zeta_{1}=8\|\| L\left\|\left.\right|^{2}\left(\frac{2}{\eta}\|P\|+1\right)\right\| P \| \max _{\mu \in\left[\tau_{\min }, \tau_{\max }\right]}\left(\left\|e^{\bar{A} \mu}\right\|^{2}\right), \\
\zeta_{2}=2\|\| \Gamma-L\left\|\left.\right|^{2}\left(\frac{2}{\eta}\|P\|+1\right)\right\| P\left\|\max _{\mu \in\left[\tau_{\min }, \tau_{\max }\right]}\left(\left\|e^{\bar{A} \mu}\right\|^{2}\right)\right\| \xi_{\text {ueq }} \|^{2} .
\end{gathered}
$$

Now, similarly to [3, Proposition 3.29 ], and using Lemma 1, the solution $\phi$ verifies the inequality (18). Thus, the hybrid system (13)-(14) is ISS with respect to $v(t, j)$ relatively to the set $\mathscr{A}$. Finally, the interval observer (4), (5), (8) for the system (2)-(3) has ISS estimation error w.r.t $v(t, j)$ relatively to the set $\mathscr{A}$, and this concludes the proof. 
Remark 5. Note that assuming (19) to hold implies that the eigenvalues of $e^{\bar{A} \mu} \Gamma(L)$ are strictly contained in the unit circle for every $\mu$ belonging to $\left[\tau_{\min }, \tau_{\max }\right]$. Contrariwise to [4, Remark 3], it is not straightforward to connect this condition with a detectability property mainly because of the decomposition of $I+L C$ into matrix $\Gamma(L)$. However, one may conjecture that at least the following two necessary conditions are required for the feasibility of inequality (19):

(i) The detectability of the equivalent discrete-time system (DTS) of (2) given as: $\chi_{k+1}=e^{A \tau} \chi_{k}$, $y_{k}=C \chi_{k} \forall \tau \in\left[\tau_{\min }, \tau_{\max }\right]$, where $\chi$ is the state and $y_{\chi}$ is the output. Similar to the criteria given in [20, Remark 7], [4], [11], the criterion should be the detectability of the pair $\left(e^{A \tau}, C\right)$ for every $\tau \in\left[\tau_{\min }, \tau_{\max }\right]$.

(ii) The second condition is due to our use of the IPR. In general, the stability of the above equivalent DTS does not imply the stability of its IPR. However, we can use the result of [13] on the relation between the eigenvalues of a system and the stability of its IPR. In [13], they have showed that the IPR of a system is stable if its eigenvalues remain in a restricted region of the complex plane defined by $\mathscr{P}=\{z \in \mathbb{C}: \mathfrak{R e}(z)+|\mathfrak{I m}(z)|<1\}$. This condition should also be satisfied by the equivalent DTS which restricts further the synthesis feasibility.

The combination of these two conditions deserves further analysis that goes beyond the scope of the paper. It will be investigated in future work.

So far, a verification method has been given. The synthesis of the observation gain $L$ cannot be achieved using convex solvers (CS) due to the decomposition of $\left(I_{n}+L C\right)$. However, using the positive realization of this matrix, the synthesis is still possible using CS. In the following section, we propose a synthesis methodology.

\section{SYNTHESIS METHOD}

In this section, we propose a new design methodology as second contribution of this paper. We will show how to design the observer gain based on positive system theory.

\section{A. Positive realization based synthesis}

Let us now re-consider the generic impulsive correction step of (7). Let us denote $G=I+L C$, and introduce $G^{+}$and $G^{-}$, the positive and the negative part of the matrix $G$, respectively, i.e., 
$G=G^{+}-G^{-}$, equation (7) can be rewritten as

$$
\begin{aligned}
x^{+} & =G x+L(v-y) \\
& =\left(G^{+}-G^{-}\right) x+L(v-y),
\end{aligned}
$$

Let us note that for any two positive matrices $G_{1}, G_{2} \in \mathbb{R}_{\geq}^{n \times n}$ that satisfy $G=G_{1}-G_{2}$ there exists $\Delta \in \mathbb{R}_{\geq}^{n \times n}$ such that

$$
G=\left(G^{+}+\Delta\right)-\left(G^{-}+\Delta\right)
$$

That is, matrices $G_{1}$ and $G_{2}$ are any positive realization of the matrices $G^{+}$and $G^{-}$, respectively. Under the positive realization of the reset matrix $G$, the reset equation of the estimation error (9) can be generalized by the following difference equation

$$
\left[\begin{array}{l}
\underline{e}^{+} \\
\bar{e}^{+}
\end{array}\right]=\Gamma\left(G_{1}, G_{2}\right)\left[\begin{array}{l}
\underline{e} \\
\bar{e}
\end{array}\right]+\Upsilon
$$

where

$$
\Gamma\left(G_{1}, G_{2}\right)=\left[\begin{array}{ll}
G_{1} & G_{2} \\
G_{2} & G_{1}
\end{array}\right]
$$

Therefore, the idea for the synthesis is to calculate numerically the two positive matrices $G_{1}$ and $G_{2}$ that satisfy the stability conditions. Then, one can compute directly the matrices $G^{+}$and $G^{-}$ as the positive and the negative parts of the matrix $G$ obtained from $G=G_{1}-G_{2}$. Using (33) instead of $\Gamma(L)$ in inequality (19), and introducing

$$
\Phi\left(P, G_{1}, G_{2}, L\right)=\Gamma\left(G_{1}, G_{2}\right)^{\top} e^{\bar{A}^{\top} \mu} P e^{\bar{A} \mu} \Gamma\left(G_{1}, G_{2}\right)-P,
$$

the gain synthesis can now be performed by finding solution $\left\{P, G_{1}, G_{2}, L\right\}$ to the following feasibility problem

$$
\begin{aligned}
& \Phi\left(P, G_{1}, G_{2}, L\right) \prec 0, \quad \forall \mu \in\left[\tau_{\min }, \tau_{\max }\right], \\
& I_{n}+L C=G_{1}-G_{2}, \\
& G_{1} \geq 0, \\
& G_{2} \geq 0, \\
& P \succ 0
\end{aligned}
$$


From equation (31) and based on the definition of the positive matrices $G^{+}$and $G^{-}$and their positive realization $G_{1}$ and $G_{2}$, respectively, the reset equation (32) can be seen as a positive reset $\Gamma(L)$ perturbed by a nonnegative matrix as follows

$$
\Gamma\left(G_{1}, G_{2}\right)=\Gamma(L)+\mathbb{1}_{2 \times 2} \otimes \Delta
$$

Remark 6. Since the matrix $\Delta$ is nonnegative which implies that $\mathbb{1}_{2 \times 2} \otimes \Delta$ is also nonnegative, it is always possible to enhance the interval observer dynamics at jumps in (32) by reducing the matrix $\Gamma\left(G_{1}, G_{2}\right)$ in (36) to its optimal realization $\Gamma(L)$. As a result, the observer gain matrix $L$ in equations (8) of the interval observer can be synthesized using the intermediate auxiliary matrices $G_{1}$ and $G_{2}$, then the implementation is finally done using matrix $\Gamma(L)$ instead of $\Gamma\left(G_{1}, G_{2}\right)$.

\section{B. Design procedure}

The semi-definite programming (SDP) (35) is subjected to a Nonlinear Matrix inequality, which is hard to solve. The constraint $\Phi \prec 0$ can be relaxed to a Bilinear Matrix Inequality (BMI) using the simplifications below.

The semi-definite constraints to be simplified are as follows

$$
\begin{array}{r}
\Phi\left(P, G_{1}, G_{2}, L\right) \prec 0 \\
-P \prec 0
\end{array}
$$

these constraints can be combined by using the projection lemma 2 .

Lemma 2. (Projection Lemma [21]) Given $Z=Z^{\top} \in \mathbb{R}^{m \times m}$ and two matrices $U$ and $V$ of column dimension $m$; there exists an unstructured matrix $F$ that satisfies

$$
U^{\top} F V+V^{\top} F^{\top} U+Z \prec 0
$$

if and only if the following projection inequalities with respect to $F$ are satisfied

$$
\begin{aligned}
& N_{U}^{\top} Z N_{U} \prec 0, \\
& N_{V}^{\top} Z N_{V} \prec 0,
\end{aligned}
$$

where $N_{U}$ and $N_{V}$ are arbitrary matrices which columns form a basis of the nullspaces of $U$ and $V$, respectively.

Setting

$$
Z=\left[\begin{array}{cc}
e^{\bar{A}^{\top} \mu} P e^{\bar{A} \mu} & 0 \\
0 & -P
\end{array}\right]
$$


Thus, the equivalence between (39) and (37) is obtained with $N_{U}^{\top}=\left[\Gamma\left(G_{1}, G_{2}\right)^{\top} \quad I_{2 n}\right]$ and $N_{V}^{\top}=\left[\begin{array}{ll}0 & I_{2 n}\end{array}\right]$. Picking $U=\left[\begin{array}{ll}-I_{2 n} & \Gamma\left(G_{1}, G_{2}\right)\end{array}\right]$ and $V=\left[\begin{array}{ll}I_{2 n} & 0\end{array}\right]$ that have $N_{U}$ and $N_{V}$ as nullspace basis, respectively, the inequalities (37) can be rewritten in the form (38) as follows

$$
\left[\begin{array}{cc}
e^{\bar{A}^{\top} \mu} P e^{\bar{A} \mu}-F-F^{\top} & F \Gamma\left(G_{1}, G_{2}\right) \\
\star & -P
\end{array}\right] \prec 0
$$

Using Schur complement for the inequality (41) we have

$$
\left[\begin{array}{ccc}
-F-F^{\top} & F \Gamma\left(G_{1}, G_{2}\right) & e^{\bar{A}^{\top} \mu} \\
\star & -P & \mathbf{0} \\
\star & \star & -P^{-1}
\end{array}\right] \prec 0
$$

Pre-and-post multiplying (42) by the matrix $\operatorname{diag}\left\{I_{2 n}, I_{2 n}, P\right\}$ on gets

$$
\left[\begin{array}{ccc}
-F-F^{\top} & F \Gamma\left(G_{1}, G_{2}\right) & e^{\bar{A}^{\top} \mu} P \\
\star & -P & \mathbf{0} \\
\star & \star & -P
\end{array}\right] \prec 0
$$

In the inequality (43) the time variable $\mu$ is in a compact interval defined as $\mu \in\left[\tau_{\min }, \tau_{\max }\right]$. So the term $e^{\bar{A} \mu}$ can be represented by its polytopic over-approximation. This over-approximation consists in determining a finite number of constant matrices $\left\{M_{1}, M_{2}, \ldots, M_{v}\right\} \in \mathbb{R}^{2 n \times 2 n}$ such that $e^{\bar{A}\left[\tau_{\min }, \tau_{\max }\right]} \in \operatorname{conv}\left\{M_{1}, M_{2}, \ldots, M_{v}\right\}$. There exist several methods in the literature for determining the matrices $\left\{M_{i}\right\}_{i \in\{1, \ldots, v\}}$ [22]. Here, we use the method based on Taylor's series developed in [23].

Finally, to solve the matrix inequalities (37), it is sufficient to solve the set of BMIs given as follows

$$
\left[\begin{array}{ccc}
-F-F^{\top} & F \Gamma\left(G_{1}, G_{2}\right) & M_{i}^{\top} P \\
\star & -P & \mathbf{0} \\
\star & \star & -P
\end{array}\right] \prec 0 \quad \forall i \in\{1, \ldots, v\}
$$

It is worth pointing that the matrices $\left\{P, G_{1}, G_{2}, L\right\}$ are the decision variables in this set of BMIs.

The second result of this paper is the design of the observer gain, which is summarized in the following proposition.

Proposition 1. Let Assumptions 1, 2, 3, and 4 hold. If there exist two nonnegative matrices $G_{1}$, $G_{2} \in \mathbb{R}^{n \times n}$, and a matrix $L \in \mathbb{R}^{n \times p}$ such that the set of BMIs (44) and the constraints (35b)-(35d) 
are feasible, then the interval observer of the form (4), (5) and (8) for the system (respectively, noise-free system) (2)-(3) has an ISS (respectively, a GES) estimation error w.r.t. the set $\mathscr{A}$.

Proof of Proposition 1. The proof is given in the previous lines both by applying the positive realization and the projection lemma.

\section{ILLUSTRATIVE EXAMPLES}

In order to illustrate the performance of the proposed observer, we consider a Spring-massdamper system.

Let $p, \kappa_{v}$, and $F$ be the position, the velocity, and the force applied to the object, respectively. We have

$$
\begin{aligned}
\dot{p}(t) & =\kappa_{v}(t)+d_{1}(t) \\
m_{s} \dot{\kappa}_{v}(t) & =-k_{s} p(t)-c_{s} \kappa_{v}(t)+F(t)+d_{2}(t)
\end{aligned}
$$

where $m_{s}, k_{s}$ and $c_{s}$ stand for the mass of the object, the stiffness constant of the spring, and the damping ratio, respectively, and $d(t)=\left[d_{1}(t) d_{2}(t)\right]^{\top}$ is the state disturbance. Defining $x_{1}=p$, $x_{2}=\kappa_{v}$, and $u=F$, with the practical parameters of the system which are given as $m_{s}=1 \mathrm{~kg}$, $k_{s}=1 \mathrm{~N} / \mathrm{m}$ and $c_{s}=1 \mathrm{Ns} / \mathrm{m}$, one can introduce its LTI model as

$$
\dot{x}=\left[\begin{array}{cc}
0 & 1 \\
-1 & -1
\end{array}\right] x+\left[\begin{array}{l}
0 \\
1
\end{array}\right] u+d(t)
$$

where $d(t)$ is unknown-but-bounded as $-\bar{d} \leq d(t) \leq \bar{d}$ such that $\bar{d}=\left[\begin{array}{ll}1 & 1\end{array}\right]^{\top}$, and the output is given by $y\left(t_{k}\right)=2 x_{1}\left(t_{k}\right)+v\left(t_{k}\right)$ where $v\left(t_{k}\right)=0.4 \cos \left(2 t_{k}\right)$. The system is not cooperative. So the solution that we have proposed is to use its IPR. Then, the dynamics of the interval estimation error between two consecutive measurements in (6) is defined by the following matrices $A^{M}=$ $\left[\begin{array}{cc}0 & 1 \\ 0 & -1\end{array}\right], A^{N}=\left[\begin{array}{ll}0 & 0 \\ 1 & 0\end{array}\right]$. Note that although the plant model is stable, the observer dynamics in between measurements is unstable. To illustrate the performance of the observer, we fix $c_{s}=1$ and we choose the time interval $\left[\tau_{\min }, \tau_{\max }\right]=[0.04,0.3]$, then we solve the feasibility problem given in Proposition 1. The set of BMIs (44) is solved using the YALMIP toolbox [24] based on the Penlab (v1.04) solver. The observation gain matrix is obtained as $L^{\top}=[-0.5004-0.0494]$. The implementation is made with matrix $\left(I_{2}+L C\right)=G_{1}-G_{2}=\left[\begin{array}{cc}-0.0008 & 0 \\ -0.0987 & 1\end{array}\right]$. The measurement times are generated randomly in the time interval $\left[\tau_{\min }, \tau_{\max }\right]$. The system input in taken as 

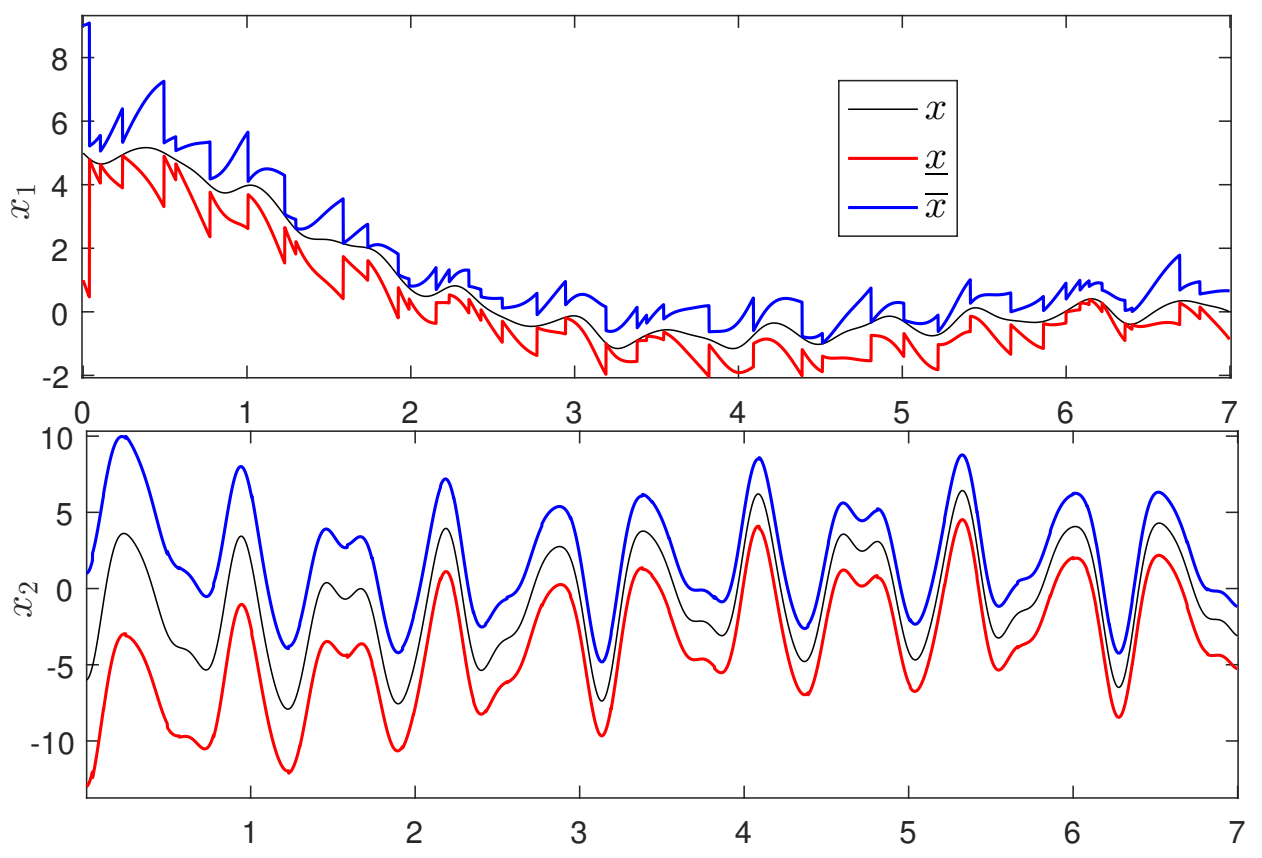

Figure 1. Simulation results for the spring-mass-damper system: the estimate bounds for the position (top), and velocity (bottom).

$u(t)=10[2 \sin (10 t)+\sin (16 t)+\sin (24 t)]$. The simulation results are given in Figure 1 and 2. In Figure 1, it is noticeable that the jump part of the interval impulsive observer maintains the estimate bounds from diverging, even though the open-loop dynamics of the interval estimator error is unstable. In Figure 2, the estimates converge exponentially to the state, and the Lyapunov function of the error shows a faster convergence at jumps while stays almost constant when flowing.

In order to show the maximum allowable inter-measurement time on the problem feasibility, we vary the damping ratio $c_{s}$ which in turn varies the eigenvalues of the flow part. The maximum inter-measurement time $\tau_{\max }$ that renders the design problem feasible, for different values of $c_{s}$, is given in Table I. One can notice that the feasibility depends on the maximum inter-measurement

Table I

The relation between $\tau_{\max }$ and the damping ratio.

\begin{tabular}{|c|c|c|c|c|c|c|c|c|}
\hline$c_{s}$ & 1 & 0.9 & 0.8 & 0.7 & 0.6 & 0.5 & 0.4 & 0.3 \\
\hline$\tau_{\max }$ & 1.1 & 1 & 0.9 & 0.8 & 0.5 & 0.3 & 0.05 & - \\
\hline
\end{tabular}




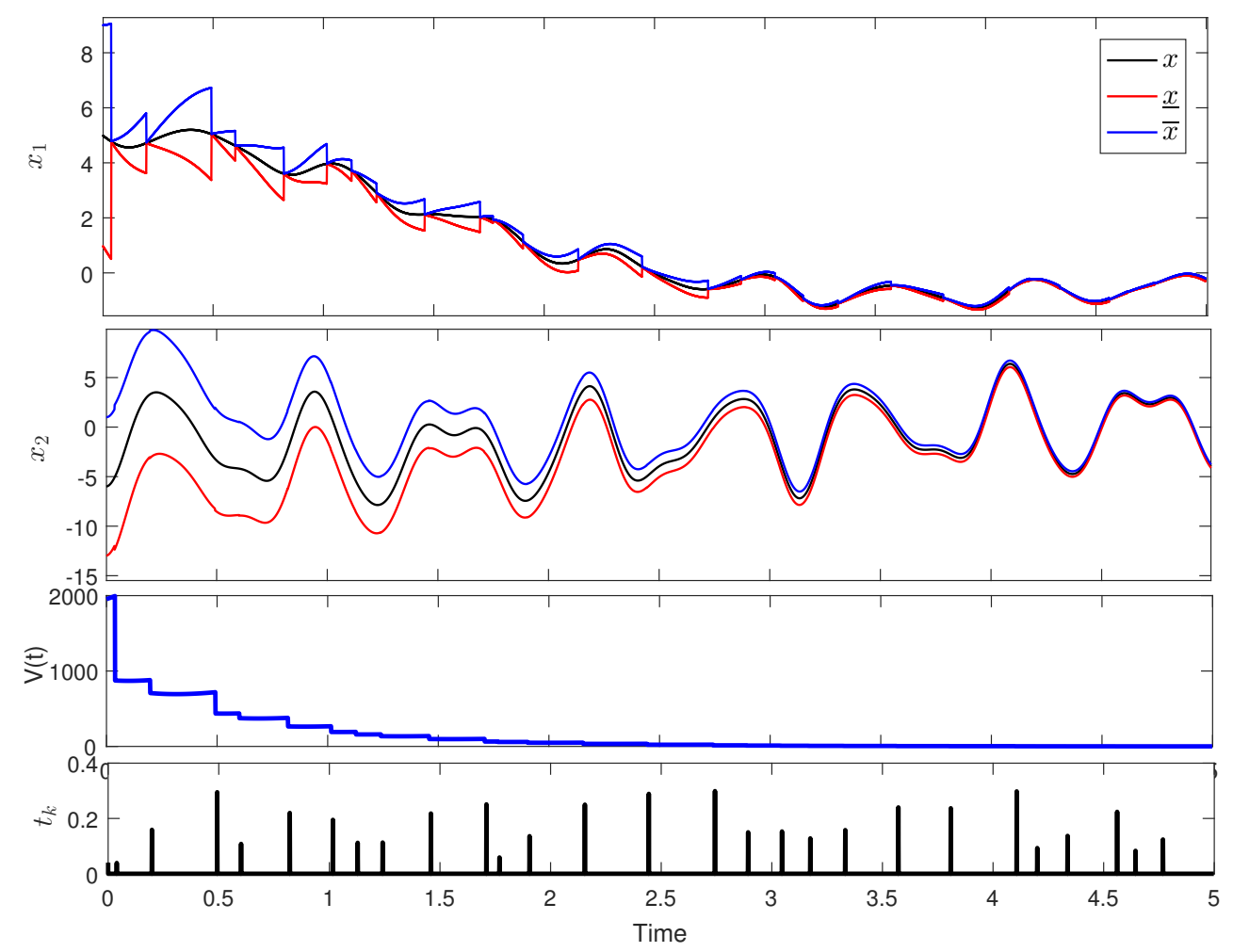

Figure 2. System without perturbations and noises : from top to bottom; state $x_{1}, x_{2}$, Lyapunov function of the error, and the inter-measurement times.

time $\tau_{\max }$. The correction jumps are made to maintain the prediction from diverging, so the larger the divergence rate of the prediction error, the smaller the feasible $\tau_{\text {max }}$. For damping ratio $c_{S} \leq 0.3$, it was not possible to find a gain $L$ that renders the eigenvalues of the discrete system equivalent (see Remark 5) in the restricted region of the complex plane given in [13]. Such an issue is the main source of conservatism of the approach proposed in the paper.

\section{CONCLUSIONS}

In the paper, a new approach for designing interval impulsive observers for linear systems with aperiodic discrete measurement has been introduced. Exploring the internal positivity of the system, a new method has been proposed for the design of the observer gain. The synthesis of the observation gain is performed using BMIs. Simulation results illustrate the efficiency of the proposed interval impulsive observer for a class of linear systems. Future works may focus on; (i) the co-design of the observation gain and the time interval where the measurements can interfere, (ii) the design of stabilizing control law based on interval impulsive observers. 


\section{REFERENCES}

[1] R. Postoyan and D. Nesic, "A framework for the observer design for networked control systems," IEEE Trans on Automat Contr, vol. 57, no. 5, pp. 1309-1314, 2012.

[2] J. P. Hespanha, P. Naghshtabrizi, and Y. Xu, "A survey of recent results in networked control systems," Proc. of the IEEE, vol. 95, no. 1, pp. 138-162, 2007.

[3] R. Goebel, R. G. Sanfelice, and A. R. Teel, Hybrid Dynamical Systems: modeling, stability, and robustness. Princeton University Press, 2012.

[4] F. Ferrante, F. Gouaisbaut, R. G. Sanfelice, and S. Tarbouriech, "State estimation of linear systems in the presence of sporadic measurements," Automatica, vol. 73, pp. 101-109, 2016.

[5] D. Efimov, E. Fridman, A. Polyakov, W. Perruquetti, and J.-P. Richard, "On design of interval observers with sampled measurement," Systems \& Control Letters, vol. 96, pp. 158-164, 2016.

[6] J. Gouzé, A. Rapaport, and Z. M. Hadj-Sadok, "Interval observers for uncertain biological systems," Journal of Ecological Modelling, vol. 133, pp. 45-56, 2000.

[7] D. Efimov and T. Raïssi, "Design of interval observers for uncertain dynamical systems," Automation and Remote Control, vol. 77, no. 2, pp. 191-225, 2016.

[8] G. Goffaux, A. V. Wouwer, and O. Bernard, "Improving continuous-discrete interval observers with application to microalgae-based bioprocesses," Journal of Process Control, vol. 19, no. 7, pp. 1182-1190, 2009.

[9] T. Alamo, J. Bravo, and E. Camacho, "Guaranteed state estimation by zonotopes," Automatica, vol. 41, pp. 1035-1043, 2005.

[10] N. Meslem, N. Ramdani, and Y. Candau, "Using hybrid automata for set-membership state estimation with uncertain nonlinear continuous-time systems," Journal of Process Control, vol. 20, no. 4, pp. 481-489, 2010.

[11] F. Mazenc and T. N. Dinh, "Construction of interval observers for continuous-time systems with discrete measurements," Automatica, vol. 50, no. 10, pp. 2555-2560, 2014.

[12] N. Meslem and N. Ramdani, "Interval observer design based on nonlinear hybridization and practical stability analysis," International Journal of Adaptive Control and Signal Processing, vol. 25, no. 3, pp. 228-248, 2011.

[13] F. Cacace, A. Germani, and C. Manes, "A new approach to design interval observers for 
linear systems." IEEE Trans. Automat. Contr., vol. 60, no. 6, pp. 1665-1670, 2015.

[14] L. Farina and S. Rinaldi, Positive Linear Systems: Theory and Applications. John Wiley \& Sons, 2000.

[15] J. K. Scott and P. I. Barton, "Bounds on the reachable sets of nonlinear control systems," Automatica, vol. 49, no. 1, pp. 93 - 100, 2013.

[16] H. Khalil, Nonlinear Systems. Prentice Hall, 2002.

[17] M. Müller, "Uber das Fundamentaltheorem in der Theorie der gewöhnlichen Differentialgleichungen," Mathematische Zeitschrift, vol. 26, pp. 619-645, 1927.

[18] A. R. Teel, F. Forni, and L. Zaccarian, "Lyapunov-based sufficient conditions for exponential stability in hybrid systems," IEEE Trans on Automat Contr, vol. 58, no. 6, pp. 1591-1596, 2013.

[19] C. Cai and A. R. Teel, "Characterizations of input-to-state stability for hybrid systems," Systems \& Control Letters, vol. 58, no. 1, pp. 47-53, 2009.

[20] T. Raff and F. Allgower, "Observers with impulsive dynamical behavior for linear and nonlinear continuous-time systems," in CDC. IEEE, 2007, pp. 4287-4292.

[21] G. Pipeleers, B. Demeulenaere, J. Swevers, and L. Vandenberghe, "Extended LMI characterizations for stability and performance of linear systems," Systems \& Control Letters, vol. 58, no. 7, pp. 510-518, 2009.

[22] W. P. Heemels, N. Van De Wouw, R. H. Gielen, M. Donkers, L. Hetel, S. Olaru, M. Lazar, J. Daafouz, and S. Niculescu, "Comparison of overapproximation methods for stability analysis of networked control systems," in Proc. of the HSCC Conf. ACM, 2010, pp. 181-190.

[23] L. Hetel, J. Daafouz, and C. Iung, "LMI control design for a class of exponential uncertain systems with application to network controlled switched systems," in ACC'07. IEEE, 2007, pp. 1401-1406.

[24] J. Lofberg, "YALMIP: A toolbox for modeling and optimization in MATLAB," in Proc. of the CACSD Conf. IEEE, 2004, pp. 284-289. 